\title{
Association of gene variants with longitudinal changes in high density lipoprotein cholesterol among Tehranian people: A latent growth curve model
}

\author{
Mahdi Akbarzadeh ${ }^{1}$, Abbas Moghimbeigii* Nathan Morris ${ }^{3}$, Maryam S Daneshpour ${ }^{4}$, \\ Ali Reza Soltanian ${ }^{5}$ and Hossein Mahjub ${ }^{6}$ \\ ${ }^{1}$ Ph.D Candidate in Biostatistics, Department of Biostatistics, School of Public Health, Hamadan University
} of Medical Sciences, Hamadan, Iran

${ }^{2}$ Associate Professor of Biostatistics, Modeling of Noncommunicable Disease Research Canter, Department of Biostatistics, School of Public Health, Hamadan University of Medical Sciences, Hamadan, Iran ${ }^{3}$ Associate Professor of Biostatistics, Department of Epidemiology and Biostatistics, Case Western Reserve University, Cleveland, $\mathrm{OH}, \mathrm{USA}$

${ }^{4}$ Cellular and Molecular Endocrine Research Center, Research Institute for Endocrine Sciences, Shahid Beheshti University of Medical Sciences; Tehran, Iran

${ }^{5}$ Associate Professor of Biostatistics, Modeling of Noncommunicable Disease Research Canter, Department of Biostatistics, School of Public Health, Hamadan University of Medical Sciences, Hamadan, Iran ${ }^{6}$ Professor of Biostatistics, Research Center for Health Sciences and Department of Biostatistics, School of Public Health, Hamadan University of Medical Sciences, Hamadan, Iran

\begin{abstract}
High density lipoprotein cholesterol (HDL-C) particles remove fat molecules from cells which need to export fat molecules. The aim of this study was to assess the association of SNP related to fat mass and obesity associated gene (FTO) with HDL-C change in a subset of participated families in Tehran Lipid and Glucose Study (TLGS). In this study 914 individual (with age $>3$ ) including 126 families with size $8.49 \pm 3.10$ were selected from participants in TLGS. Genomic DNA was extracted from peripheral blood using standard salting-out method. HDL-C measured in four time point from March 1999 to December 2011 with a 3-year follow-up period. We examine the association via a latent growth curve model. To achieve the study target we used the latent growth curve model (LGCM) in R in TLGS family data. Adjusted association by sex and age between some FTO genes and changes of HDL-C overtime are significant. Our findings provide basis for searching for genes affecting change in HDL-C.
\end{abstract}

KEY WORDS: HIGH DENSITY LIPOPROTEIN CHOLESTEROL, GENETIC INFLUENCE, LATENT GROWTH CURVE

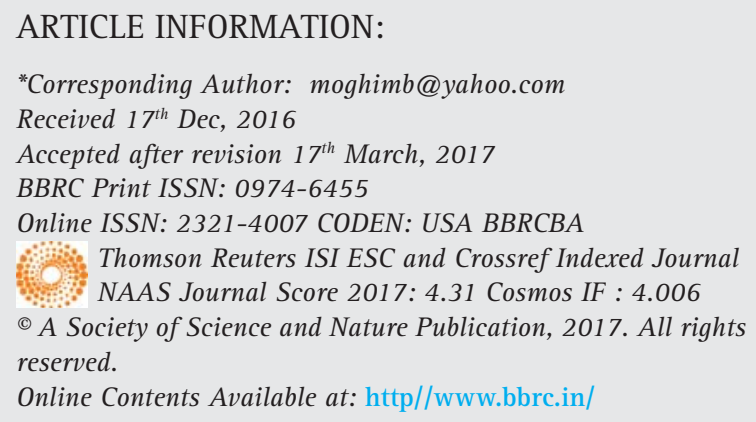




\section{INTRODUCTION}

High-density lipoproteins (HDL) are one of the five major groups of lipoproteins. Lipoproteins are complex particles composed of multiple proteins which transport all fat molecules (lipids) around the body within the water outside cells. They are typically composed of 80-100 proteins per particle and transporting up to hundreds of fat molecules per particle. Unlike the larger lipoprotein particles which deliver fat molecules to cells, HDL particles remove fat molecules from cells which need to export fat molecules. The fats carried include cholesterol, phospholipids, and triglycerides; amounts of each are quite variable. Increasing concentrations of HDL particles are strongly associated with decreasing accumulation of atherosclerosis within the walls of arteries. This is important because atherosclerosis eventually results in sudden plaque ruptures, cardiovascular disease, stroke and other vascular diseases. HDL particles are sometimes referred to as "good cholesterol" because they can transport fat molecules out of artery walls, reduce macrophage accumulation, and thus help prevent or even regress atherosclerosis. However, studies have shown that HDL-lacking mice still have the ability to transport cholesterol to bile, suggesting that there are alternative mechanisms for cholesterol removal. Also heritability of low HDL-C is demonstrated, (Asselbergs et al. 2012). Qureshi et al. have shown FTO genes have association with HDL-C in Pakistani people, (Qureshi et al. 2016).

In both epidemiological and clinical studies, as well as the meta-analyses thereof, low plasma levels of highdensity lipoprotein (HDL) cholesterol (HDL-C) identified individuals at increased risk of major coronary events. In line with a causally protective effect, HDLs exert a broad spectrum of potentially anti-atherogenic properties. Moreover, atherosclerosis was decreased or even reverted in several animal models by transgenic overexpression or exogenous application of apolipoprotein (apoA-I) the most abundant protein of HDL, (Zhang et al. 2008).

However, to date, drugs increasing HDL-C, such as fibrates, niacin, and inhibitors of cholesteryl ester transfer protein (CETP), have failed to consistently and significantly reduce the risk of major cardiovascular events, especially when combined with statins. Moreover, mutations in several human genes as well as targeting of several murine genes modulate HDL-C levels without changing cardiovascular risk and atherosclerotic plaque load, respectively, in the opposite direction as expected from the inverse correlation of HDL-C levels and cardiovascular risk in epidemiological studies. Because of these controversial data, the pathogenic role and, hence, suitability of HDL as a therapeutic target has been increasingly questioned. Because of the frequent confounding of low HDL-C with hypertriglyceridemia, it has been argued that low HDL-C is an innocent bystander of (postprandial) hypertriglyceridemia or another culprit related to insulin resistance or inflammation, (Gordon et al. 1977; Silventoinen et al. 2007). Genetic association of HDL-C is demonstrated in some population,(Kim Et Lee 2013; Nirengi et al. 2016; Bandarian et al. 2016).

In the Framingham study, high density lipoprotein is shown as a protective factor against coronary heart disease,(Gordon et al. 1977). In some studies on Iranian population, low HDL-C is reported as a risk factor of coronary artery disease(Sharifi et al. 2009; Sharifi et al. 2008; Hatmi et al. 2007).Also Akbarzadeh et al. have shown some genes association with HDL-C in the Iranian people without any results about the trend of HDL-C(Akbarzadeh et al. 2011; Alavi Majd et al. 2010). Among Iranian people as well as Tehranian people the topic has been proven (Zarkesh et al. 2012).

An advanced, powerful and flexible framework to model the latent variables is structural equation modeling (SEM). In recent years, in genetic analysis of longitudinal family data, SEM has made significant progress. One of these models is Morris and his colleagues that it would be in addition to the relationships between variables over time, taking into account the simultaneous equations to analyze genetic family data(Morris et al. 2010). The model can be able to run a wide range of genetic models with latent variables under SEM, and in recent months the model $\mathrm{R}$ package, strum, has been released(Song et al. 2015).

According to our knowledge, up to this point about the relationship between HDL-C changes related genes in Iran has not been investigated. The aim of this study was to assess the association of SNP in the gene fat mass and HDL-C-associated gene (FTO) with HDL-C change in a subset of participated families in Tehran Lipid and Glucose Study (TLGS). To achieve this target we used the latent growth curve model (LGCM) in STRUM R package in TLGS family data.

\section{MATERIAL AND METHODS}

Population and sample: The TLGS is an ongoing longitudinal large-scale community-based study, with a 3-year follow-up period, designed to estimate the prevalence of non-communicable disorders (NCD) and included a representative sample of residents of 13 districts of Tehran, capital of Iran. The TLGS has been implemented in a multistage stratified (district) cluster (families) random sampling technique, to select more than 15000 people aged > 3 years, from March 1999 to December 2011.The phases II, III, and IV were prospective follow-up studies 


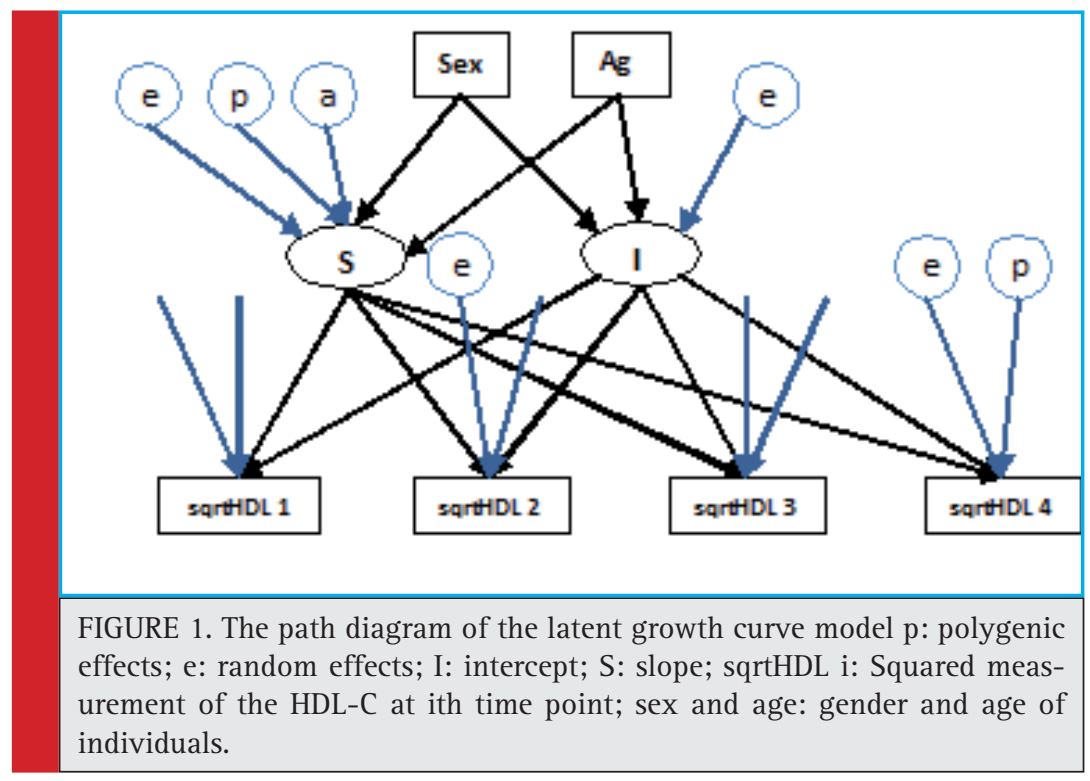

and were performed from 2002 to 2004, 2006 to 2008, and 2009 to 2011, respectively, (Azizi et al. 2002; Azizi et al. 2009). A total of 14761 individuals (valid case) were selected from the total participating cases in TLGS, including 3980 families, with an average number of 3.38 individuals, among the phase I (baseline).

This family-based study was conducted on families who participated in TLGS, with at least two members affected with syndrome metabolic (Mets).The design of TLGS includes two major components, a cross-sectional prevalence study of CVD and associated risk factors and a prospective 20-year follow up in five phases(14). In the study 914 individual including 126 families with size $8.49 \pm 3.10$ person from participated people in TLGS. Participants provided informed consent and the study was approved by the institutional ethics committees of the Research Institute for Endocrine Sciences affiliated to Shahid Beheshti University of Medical Sciences, Tehran, Iran. Information on age, sex, demographic and medication usage for treatment diabetes, hypertension and lipid disorders were collected with a standardized questionnaire.

SNP Selection and Typing: Genomic DNA was extracted from peripheral blood using standard saltingout method(Koshy et al. 2016). 5 selected polymorphisms (rs708272, rs1864163, rs1558902, rs7202116, rs9939609) studied by Tetra ARMS method, by determining through the NCBI site. MAF among for the five markers is minimum 0.039 and maximum 0.363 . Which is as follows: Our T-ARMS assay with different inner allele specific primers to produce allele-specific PCR products. Two outer primers will produce a PCR product to be used as an internal control for reaction. For all studied SNPs, the PCR reaction was optimized in a $12.5 \mu \mathrm{l}$ total vol- ume containing $1.5 \mu$ DNA template, $6.25 \mu$ l Master Mix containing ( $\mathrm{MgCl} 2$, Smart Taq polymerase (Cinna Gene Co; Iran) and BSA 0.1\% (TaKaRa; Japan) and $2 \mu \mathrm{l}$ primer containing (outers and inners ) and $2.75 \mu \mathrm{l}$ water.

Details of the primers information and final fragments are mentioned in table 1 . The optimized thermo cycling conditions were as follows: $5 \mathrm{~min}$ at $95^{\circ} \mathrm{C} ; 10$ cycles of $45 \mathrm{sec}$ at $94^{\circ} \mathrm{C}, 30 \mathrm{sec}$ at $63^{\circ} \mathrm{C}$, and $35 \mathrm{sec}$ at $72^{\circ} \mathrm{C}$; followed by 25 cycles of $45 \mathrm{sec}$ at $94^{\circ} \mathrm{C}, 30 \mathrm{sec}$ at $61^{\circ} \mathrm{C}$, and $30 \mathrm{sec}$ at $72^{\circ} \mathrm{C}$; and final extension at $72^{\circ} \mathrm{C}$ for 5 minutes. When the PCR products were separated by size via agarose gel electrophoresis, each genotype generated a special band. Accuracy of results was confirmed by direct sequencing of 10\% samples using outer primers.

Statistical Model: An advanced, powerful and flexible framework to model the latent variables is structural equation modeling (SEM).Structural equation modeling evaluates the relationships among manifest variables, manifest-latent variables and latent variables. Moreover the latent growth curve modeling (LGCM) has been recently developed in longitudinal study (Bollen\&t Curran 2006). One of advantage of the model is that allows for individual changes to be analyzed in the SEM framework and includes additional latent factors in the linear model for testing a linear, quadratic, cubic, or spline growth trajectory (Xitao \&t Xiaotao 2005).One advantage of this model compared to the routine analytical methods for longitudinal data is that modeling latent variables over time and also the intercept and slop modeled as latent variables.

The recent presented SEM is capable to model a broad relationships between latent variables based linkage or association analysis. In strum a novel score test developed. This method is a computationally rapid test of association with many SNPs 
in GWAS data (Song et al. 2015). In this new score test, we first assessed the null model fit without any SNPs in the model to confirm the appropriateness of the model for the data. In the research, HDL-C measured in 4 time point. We examined association of 5 markers of FTO genes with change of HDL-C in Tehranian pedigrees using strum $\mathrm{R}$ package.

The graphical representations of analysis model with latent variable for longitudinal HDL-C are shown for the latent growth curve model in figure 1. Variable sqrtHDL$C$ is the main trait values corrected for the use of normality. Nodes marked with: "p" are polygenic effects, and "e" are random effects. "I" is the intercept and S is the slope.

After removing the SNPs with no variation in the data set or with no score test results, the remaining 18 SNPs were tested for association with the main trait by coding additively as 0,1 , or 2 based on the minor allele count.

\section{RESULTS AND DISCUSSION}

The genomic inflation factors were 1.01 and 1.02 for each model. The MAF (minor allele frequency) of all SNPs are greater than 5\% and they are informative for association analysis. Also the CFI (comparative fit index) of the conceptual model was 99\% shown that the model had good fitness. The association between 5 FT0 markers and slop of HDL-C was significant (ie, $\mathrm{P}$ value $<1.0 \mathrm{e}-5$ ). Table 1 shows the characteristics and $\mathrm{P}$ values.

Changing in HDL-C in human is certainly under genetic influence(Kim \& Lee 2013; Nirengi et al. 2016; Bandarian et al. 2016). Actually, the changes of HDL-C is a latent variable. A preferable understanding way to the change discovery is measuring by natural indicator such as HDL-C. The study is the first to simultaneously analyze level and change in HDL-C using a population-based longitudinal study of individual including families. We found a siginificant genetic influence on change in HDL-C for four known markers. The modeling assumes that long-term (15 year) individual HDL-C trajectories in the four waves of young adults aged 3-83 years at baseline is adequately described by individual initial level and rate of change.

\begin{tabular}{|c|c|c|c|}
\hline SNP & $\mathrm{Al}$ & MAF & $P$ value \\
\hline rs708272 & $\mathrm{G} / T$ & 0.1232 & $3.320 \mathrm{E}-16$ \\
\hline rs 1558902 & $\mathrm{G} / T$ & 0.1102 & $2.045 \mathrm{E}-13$ \\
\hline rs7202116 & $\mathrm{C} / \mathrm{T}$ & 0.1851 & $1.489 \mathrm{E}-06$ \\
\hline rs9939609 & $\mathrm{T} / T$ & 0.1448 & $2.276 \mathrm{E}-06$ \\
\hline rs6696438 & $\mathrm{C} / \mathrm{T}$ & 0.1476 & $4.462 \mathrm{E}-06$ \\
\hline
\end{tabular}

\section{CONCLUSION}

Our findings provide basis for searching for genes affecting change in HDL-C. We assume that the genetic influence on change in HDL-C has a polygenic origin with finite known markers. However, trying to identifying genetic variants for HDL-C change may be require a whole human genome wide scan in the target population.

\section{ACKNOWLEDGMENTS}

We express appreciation to the participations and TLGS staff for their guidance in the preparing dataset. We thank the reviewers for their insightful and helpful comments.

\section{REFERENCES}

Akbarzadeh, Mahdi, H A Majd, Maryam Sadat Daneshpour, Yadolah Mehrabi, and Freydoon Azizi. (2011). Analyzing of Multivariate Two Levels Haseman-Elston Regression and Its Application in Genetic Linkage of HDL-C, Triglycerides and Waist in 91 Iranian Families with Metabolic Syndrome. Koomesh 12 (3). Semnan University of Medical Sciences and Health Services: Pe266-Pe271.

Alavi Majd, H, M Akbarzadeh, Y Mehrabi, M S Daneshpour, and F Azizi. (2010). Genetic Linkage Analysis of HDL-C in 91 Iranian Families with Metabolic Syndrome with HasemanElston Regression Methods. HBI_Journals 7 (4): 243-47.

Asselbergs, Folkert W, Yiran Guo, Erik P A van Iperen, Suthesh Sivapalaratnam, Vinicius Tragante, Matthew B Lanktree, Leslie A Lange, Berta Almoguera, Yolande E Appelman, and John Barnard. (2012). Large-Scale Gene-Centric Meta-Analysis across 32 Studies Identifies Multiple Lipid Loci. The American Journal of Human Genetics 91 (5). Elsevier: 823-38.

Azizi, Fereidoun, Arash Ghanbarian, Amir Abbas Momenan, Farzad Hadaegh, Parvin Mirmiran, Mehdi Hedayati, Yadollah Mehrabi, and Saleh Zahedi-Asl. (2009). Prevention of NonCommunicable Disease in a Population in Nutrition Transition: Tehran Lipid and Glucose Study Phase II. Trials 10 (January): 5. doi:10.1186/1745-6215-10-5.

Azizi, Fereidoun, Maziar Rahmani, Habib Emami, P Mirmiran, R Hajipour, M Madjid, J Ghanbili, (2002). Cardiovascular Risk Factors in an Iranian Urban Population: Tehran Lipid and Glucose Study (Phase 1). Sozial- Und Präventivmedizin 47 (6). Birkhäuser Verlag: 408-26. doi:10.1007/s000380200008.

Bandarian, Fatemeh, Maryam Sadat Daneshpour, Mehdi Hedayati, Mohsen Naseri, and Fereidoun Azizi. (2016). Identification of Sequence Variation in the Apolipoprotein A2 Gene and Their Relationship with Serum High-Density Lipoprotein Cholesterol Levels.Iranian Biomedical Journal 20 (2). Pasteur Institute of Iran: 84.

Bollen, KA, and PJ Curran.( 2006). Latent Curve Models: A Structural Equation Perspective. John Wiley \& Sons, Inc. 
Gordon, Tavia, William P Castelli, Marthana C Hjortland, William B Kannel, and Thomas R Dawber. (1977). High Density Lipoprotein as a Protective Factor against Coronary Heart Disease: The Framingham Study. The American Journal of Medicine 62 (5). Elsevier: 707-14.

Hatmi, Z N, S Tahvildari, A Gafarzadeh Motlag, and A Sabouri Kashani. (2007). Prevalence of Coronary Artery Disease Risk Factors in Iran: A Population Based Survey BMC Cardiovascular Disorders 7 (1). BioMed Central: 1.

Kim, Jongwoo, and Seon Yeong Lee (2013 )Obesity and HighDensity Lipoprotein Cholesterol (HDL-C): The Recent Related Research Trend Including New Generation Drugs for HDL-C The Korean Journal of Obesity 22 (2): 67-72.

Koshy, Linda, A L Anju, S Harikrishnan, V R Kutty, V T Jissa, Irin Kurikesu, Parvathy Jayachandran, A Jayakumaran Nair, A Gangaprasad, and G M Nair (2016). Evaluating Genomic DNA Extraction Methods from Human Whole Blood Using Endpoint and Real-Time PCR Assays. Molecular Biology Reports. Springer, 1-12.

Morris, Nathan J, Robert C Elston, and Catherine M Stein. (2010). A Framework for Structural Equation Models in General Pedigrees. Human Heredity 70 (4): 278-86. doi:10.1159/000322885.

Nirengi, Shinsuke, Mami Fujibayashi, Kokoro Tsuzaki, Sachiko Furuno, Akihiko Uchibe, Yasuharu Kawase, Kazuhiko Kotani, and Naoki Sakane. (2016) ACTN3 Gene R577X Polymorphism Associated with High-Density Lipoprotein Cholesterol and Adiponectin in Rugby Players. Endocrine Practice. American Association of Clinical Endocrinologists.

Qureshi, Sarah Anwar, Amir Mumtaz, and Saleem Ullah Shahid. (2016)A Common Variant, rs3751812, in Fat Mass and Obesity Associated (FT0) Gene Is Associated with Serum High and Low Density Lipoprotein Cholesterol in the Pakistani Subjects. Nutrition. Elsevier.

Sharifi, F1, S N Mousavinasab, M Saeini, and M Dinmohammadi. (2009). Prevalence of Metabolic Syndrome in an Adult
Urban Population of the West of Iran. Experimental Diabetes Research 2009. Hindawi Publishing Corporation.

Sharifi, F, S N Mousavinasab, R Soruri, M Saeini, and M Dinmohammadi (2008) High Prevalence of Low High-Density Lipoprotein Cholesterol Concentrations and Other Dyslipidemic Phenotypes in an Iranian Population Metabolic Syndrome and Related Disorders 6 (3). Mary Ann Liebert, Inc. 140 Huguenot Street, 3rd Floor New Rochelle, NY 10801-5215 USA: 187-95.

Silventoinen, Karri, Meike Bartels, Daniëlle Posthuma, G Frederiek Estourgie-van Burk, Gonneke Willemsen, Toos C E M van Beijsterveldt, and Dorret I Boomsma (2007) Genetic Regulation of Growth in Height and Weight from 3 to 12 Years of Age: A Longitudinal Study of Dutch Twin Children Twin Research and Human Genetics 10 (2). Cambridge Univ Press: 354-63. doi:https://doi.org/10.1375/twin.10.2.354.

Song, Yeunjoo E, Catherine M Stein, and Nathan J Morris. (2015) Strum: An R Package for Structural Modeling of Latent Variables for General Pedigrees.BMC Genetics 16 (1): 35. doi:10.1186/s12863-015-0190-3.

Xitao, Fan, and Fan Xiaotao (2005) Power of Latent Growth Modeling for Detecting Linear Growth: Number of Measurements and Comparison with Other Analytic Approaches. The Journal of Experimental Education 73 (2). Taylor \& Francis: 121-39.

Zarkesh, Maryam, Maryam Sadat Daneshpour, Bita Faam, Mohammad. (2012) Heritability of the Metabolic Syndrome and Its Components in the Tehran Lipid and Glucose Study (TLGS). Genetics Research 94 (6): 331-37. doi:10.1017/ S001667231200050X.

Zhang, Da-Wei, Rita Garuti, Wan-Jin Tang, Jonathan C Cohen, and Helen H Hobbs (2008) Structural Requirements for PCSK9Mediated Degradation of the Low-Density Lipoprotein Receptor Proceedings of the National Academy of Sciences 105 (35). National Acad Sciences: 13045-50. 